\title{
EXTENSIONS OF REPRESENTABLE POSITIVE LINEAR FUNCTIONALS TO UNITIZED QUASI *-ALGEBRAS
}

\author{
GIORGIA BELLOMONTE
}

\begin{abstract}
It is known that, under certain conditions, ${ }^{*}$-representability and extensibility to the unitized ${ }^{*}$-algebra of a positive linear functional, defined on a $*$-algebra without unit, are equivalent. In this paper, a new condition for an analogous result is given for the case of a hermitian linear functional defined on a quasi *-algebra $\left(\mathfrak{A}, \mathfrak{A}_{0}\right)$ without unit.
\end{abstract}

\section{INTRODUCTION}

The study of linear positive functionals on *-algebras and their *-representability has been undertaken since the 1970s. In [8], Powers proved that, if $\omega$ is a positive linear functional on a ${ }^{*}$-algebra $\mathfrak{A}$ with unit, it is possible to construct a closed strongly cyclic ${ }^{*}$-representation $\pi$ of $\mathfrak{A}$ on a Hilbert space $\mathcal{H}$, generalizing, in this way, the well known GNS construction for $\mathrm{C}^{*}$-algebras

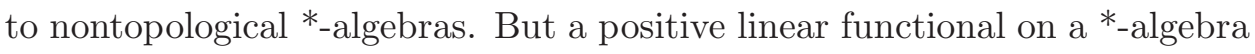
without unit does not extends automatically to a positive linear functional on the unitization.

In the 1980s, several authors (see [7] and references therein), improving [9], showed that a positive linear functional on a ${ }^{*}$-algebra $\mathfrak{A}$ without unit can be extended to a positive linear functional $\omega^{e}$ on its unitization $\mathfrak{A}^{e}=\mathfrak{A} \oplus \mathbb{C}$ if, and only if, it is hermitian, i.e., $\omega\left(a^{*}\right)=\overline{\omega(a)}, \forall a \in \mathfrak{A}$ and Hilbert bounded, i.e. there exists $\delta>0$ such that $|\omega(a)|^{2} \leq \delta \omega\left(a^{*} a\right)$, for every $a \in \mathfrak{A}$. Hence the result of Powers applies to the extension $\omega^{e}$, and then the linear positive functional $\omega$ is *-representable, too.

Contrary to the case of *-algebras, a positive linear functional defined on a quasi *-algebra $\left(\mathfrak{A}, \mathfrak{A}_{0}\right)$ with unit is not automatically representable. For this reason, if $\left(\mathfrak{A}, \mathfrak{A}_{0}\right)$ has no unit, the notion of extensibility has to be modified: a positive linear functional $\omega$ will be called extensible if it is the

1991 Mathematics Subject Classification. 46K10, 46K70, 47L60.

Key words and phrases. Representable linear functionals, unitized quasi *-algebras. 
restriction to $\left(\mathfrak{A} \oplus\{0\}, \mathfrak{A}_{0} \oplus\{0\}\right)$ of some representable positive linear functional $\omega^{e}$ defined on the unitization $\left(\mathfrak{A}^{e}, \mathfrak{A}_{0}^{e}\right)$.

Representability of a positive linear functional on a quasi *-algebra is a topical problem, see e.g. [6, 2, and it is of a certain interest in some application see, for instance, [3] and references therein. In this paper, starting from a hermitian linear functional $\omega$ defined on a quasi *algebra $\left(\mathfrak{A}, \mathfrak{A}_{0}\right)$ without unit, we study under what conditions it is possible to extend $\omega$ to a representable linear functional, defined on a quasi *-algebra with unit, possibly the whole unitization of $\left(\mathfrak{A}, \mathfrak{A}_{0}\right)$. Section 2 is devoted to recall the main definitions and results about the subject. In Section 3, once defined the extension $\omega^{e}((x, \eta)):=\omega(x)+\eta \gamma, \gamma \in \mathbb{R}^{+}$, to the unitization of $\left(\mathfrak{A}, \mathfrak{A}_{0}\right)$, we give a new condition on $\omega$ to make the extension $\omega^{e}$ to the unitized quasi *-algebra $\left(\mathfrak{A}^{e}, \mathfrak{A}_{0}^{e}\right)$ (or to a quasi *-algebra with unit contained in $\left(\mathfrak{A}^{e}, \mathfrak{A}_{0}^{e}\right)$ ) representable; this new condition is quite natural, in fact we prove that it reduces to Hilbert boundedness on $\mathfrak{A}_{0}$; moreover if $\left(\mathfrak{A}, \mathfrak{A}_{0}\right)$ has a unit, then this condition is automatically fulfilled, hence, under this condition, representability of $\omega$ and its extensibility are equivalent.

\section{DeFinitions AND KNOWN RESUlts}

Let $\mathcal{D}$ be a dense subspace of Hilbert space $\mathcal{H}$. We denote by $\mathcal{L}^{\dagger}(\mathcal{D}, \mathcal{H})$ the set of all (closable) linear operators $X$ such that $D(X)=\mathcal{D}, D\left(X^{*}\right) \supseteq \mathcal{D}$. The set $\mathcal{L}^{\dagger}(\mathcal{D}, \mathcal{H})$ is a partial *-algebra (see [1]), i.e. it is a complex vector space with respect to the usual sum $X_{1}+X_{2}$ and the scalar multiplication $\lambda X$, with a conjugate linear involution $X \rightarrow X^{\dagger}=X^{*} \uparrow \mathcal{D}$ and a distributive partial multiplication: the (weak) partial multiplication $X_{1} \square X_{2}=X_{1}^{\dagger *} X_{2}$, defined whenever $X_{2}$ is a weak right multiplier of $X_{1}$ (equivalently, $X_{1}$ is a weak left multiplier of $\left.X_{2}\right)$, that is, iff $X_{2} \mathcal{D} \subset D\left(X_{1}^{\dagger *}\right)$ and $X_{1}^{\dagger} \mathcal{D} \subset D\left(X_{2}^{*}\right)$ (we write $X_{2} \in R^{w}\left(X_{1}\right)$ or $X_{1} \in L^{w}\left(X_{2}\right)$ ). We denote with $\mathcal{L}^{\dagger}(\mathcal{D})$ the subspace of $\mathcal{L}^{\dagger}(\mathcal{D}, \mathcal{H})$ all closable operators $A$ in $\mathcal{H}$ such that $A \mathcal{D} \subseteq \mathcal{D}$, $A^{\dagger} \mathcal{D} \subseteq \mathcal{D}$. This space with this involution and the multiplication $\square$, is a *-algebra and $A_{1} \square A_{2} \xi=A_{1}\left(A_{2} \xi\right)$ for every $\xi \in \mathcal{D}[10]$.

A quasi *algebra is a couple $\left(\mathfrak{A}, \mathfrak{A}_{0}\right)$, where $\mathfrak{A}$ is a vector space with involution *, $\mathfrak{A}_{0}$ is a *algebra and a vector subspace of $\mathfrak{A}$ and $\mathfrak{A}$ is an $\mathfrak{A}_{0^{-}}$ bimodule whose module operations and involution extend those of $\mathfrak{A}_{0}$. A quasi*-algebra is said topological (resp. locally convex) if $\mathfrak{A}$ is a topological 
(resp. locally convex) vector space with topology $\tau$, with continuous involution ${ }^{*}, \mathfrak{A}_{0}$ is a dense vector subspace of $\mathfrak{A}[\tau]$ and the module operations of $\mathfrak{A}$ and involution are separately, not necessarily jointly, continuous bilinear mappings of $\mathfrak{A}_{0} \times \mathfrak{A}$ and $\mathfrak{A} \times \mathfrak{A}_{0}$ to $\mathfrak{A}$, where $\mathfrak{A}_{0}$ carries the induced topology of $\mathfrak{A}[1,10]$.

A *-representation of a quasi *-algebra $\left(\mathfrak{A}, \mathfrak{A}_{0}\right)$ is a *-homomorphism $\pi$ of $\mathfrak{A}$ into $\mathcal{L}^{\dagger}\left(\mathcal{D}_{\pi}, \mathcal{H}_{\pi}\right)$, for some pair $\left(\mathcal{D}_{\pi}, \mathcal{H}_{\pi}\right)$ with $\mathcal{D}_{\pi}$ a dense subspace of Hilbert space $\mathcal{H}_{\pi}$ [1], i.e. a linear map $\pi: \mathfrak{A} \rightarrow \mathcal{L}^{\dagger}\left(\mathcal{D}_{\pi}, \mathcal{H}_{\pi}\right)$ such that:

i) $\pi\left(x^{*}\right)=\pi(x)^{\dagger}, \quad \forall x \in \mathfrak{A}$,

ii) if $x \in \mathfrak{A}, a \in \mathfrak{A}_{0}$, then $\pi(x)$ is a weak left multiplier of $\pi(a)$ and $\pi(x) \square \pi(a)=\pi(x a)$,

iii) $\pi(a) \in \mathcal{L}^{\dagger}\left(\mathcal{D}_{\pi}\right), \forall a \in \mathfrak{A}_{0}$.

If $\left(\mathfrak{A}, \mathfrak{A}_{0}\right)$ has unit $e$, we assume $\pi(e)=\mathbb{I}$.

If $\pi$ is a ${ }^{*}$-representation of $\left(\mathfrak{A}, \mathfrak{A}_{0}\right)$ in $\mathcal{D}_{\pi}$, then $\mathcal{D}_{\pi}$ can be endowed with a locally convex topology $t_{\pi}$ defined by the family of seminorms $x \in \mathcal{D}_{\pi} \mapsto$ $\|\pi(x) \xi\|, x \in \mathfrak{A}$. Put

$$
\mathcal{D}(\tilde{\pi})=\bigcap_{x \in \mathfrak{A}} D(\overline{\pi(x)})
$$

where $\overline{\pi(x)}$ denotes the closure of the operator $\pi(x)$. Then one defines $\tilde{\pi}(x):=\overline{\pi(x)}\left\lceil\mathcal{D}(\tilde{\pi}), x \in \mathfrak{A}\right.$. The ${ }^{*}$-representation $\tilde{\pi}$ is said the closure of $\pi$. If $\mathcal{D}(\pi)=\mathcal{D}(\widetilde{\pi})$ and $\pi=\tilde{\pi}$, the representation is said to be closed. If $\pi$ is a *-representation of $\left(\mathfrak{A}, \mathfrak{A}_{0}\right)$ in $\mathcal{D}_{\pi}$, define $\mathcal{D}_{\pi^{*}}=\bigcap_{x \in \mathfrak{A}} D\left(\pi(x)^{*}\right)$, then the adjoint representation $\pi^{*}$ is such that $\pi^{*}(x)=\pi\left(x^{*}\right)^{*} \uparrow \mathcal{D}_{\pi^{*}}, \forall x \in \mathfrak{A}$. If $\pi=\pi^{*}$, then the ${ }^{*}$-representation $\pi$ is said to be self-adjoint.

A *-representation $\pi$ of $\left(\mathfrak{A}, \mathfrak{A}_{0}\right)$ is called

- cyclic if there exists a vector $\xi \in \mathcal{D}_{\pi}$ such that $\left\{\pi(a) \xi ; a \in \mathfrak{A}_{0}\right\}$ is dense in $\mathcal{H}_{\pi}$;

- strongly cyclic if there exists a vector $\xi \in \mathcal{D}_{\pi}$ such that $\{\pi(a) \xi ; a \in$ $\left.\mathfrak{A}_{0}\right\}$ is dense in $\mathcal{D}_{\pi}$ with respect to the topology $t_{\pi}$.

In [11] Trapani gave three conditions for a linear functional $\omega$, defined on a quasi *-algebra (possibly without unit), to be representable.

A linear functional $\omega$ defined on the quasi *-algebra $\left(\mathfrak{A}, \mathfrak{A}_{0}\right)$, is said to be representable if

(L.1) $\omega\left(a^{*} a\right) \geq 0, \quad \forall a \in \mathfrak{A}_{0}$;

(L.2) $\omega\left(b^{*} x^{*} a\right)=\overline{\omega\left(a^{*} x b\right)}, \quad \forall a, b \in \mathfrak{A}_{0}, \forall x \in \mathfrak{A}$; 
(L.3) $\forall x \in \mathfrak{A}$, there exists $\gamma_{x}>0$ such that

$$
\left|\omega\left(x^{*} a\right)\right| \leq \gamma_{x} \omega\left(a^{*} a\right)^{1 / 2}, \quad \forall a \in \mathfrak{A}_{0} .
$$

Let us call $\lambda_{\omega}(a), a \in \mathfrak{A}_{0}$, the coset containing $a$ in the space $\lambda_{\omega}\left(\mathfrak{A}_{0}\right):=$ $\mathfrak{A}_{0} / N_{\omega}$, where $N_{\omega}=\left\{a \in \mathfrak{A}_{0}: \omega\left(a^{*} a\right)=0\right\}$ is a left-ideal of $\mathfrak{A}_{0}$. The quotient $\lambda_{\omega}\left(\mathfrak{A}_{0}\right)$ is a pre-Hilbert space with inner product $\left\langle\lambda_{\omega}(a) \mid \lambda_{\omega}(b)\right\rangle=$ $\omega\left(b^{*} a\right), a, b \in \mathfrak{A}_{0}$. Let $\mathcal{H}_{\omega}$ be the completion of $\lambda_{\omega}\left(\mathfrak{A}_{0}\right)$. As proved in [11, Theorem 3.5 ], even if the quasi ${ }^{*}$-algebra $\left(\mathfrak{A}, \mathfrak{A}_{0}\right)$ has not unit conditions (L.1), (L.2) and (L.3) allow a generalized GNS construction for the quasi *-algebra $\left(\mathfrak{A}, \mathfrak{A}_{0}\right)$ : there exists a ${ }^{*}$-representation $\pi_{\omega}$ of $\left(\mathfrak{A}, \mathfrak{A}_{0}\right)$ such that for any $x \in \mathfrak{A}$

$$
\omega\left(b^{*} x a\right)=\left\langle\pi_{\omega}(x) \lambda_{\omega}(a) \mid \lambda_{\omega}(b)\right\rangle
$$

$\forall a, b \in \mathfrak{A}_{0}$.

If $\left(\mathfrak{A}, \mathfrak{A}_{0}\right)$ has a unit $e, \omega$ admits a closed *-representation $\widetilde{\pi}_{\omega}$ of $\left(\mathfrak{A}, \mathfrak{A}_{0}\right)$, with strongly cyclic vector $\xi_{\omega}=\lambda_{\omega}(e)$ by completing the domain.

If, in particular, $\mathfrak{A}=\mathfrak{A}_{0}$ is a ${ }^{*}$-algebra without unit, every positive linear functional $\omega$ on $\mathfrak{A}_{0}$ is ${ }^{*}$-representable in the sense that there exist a subspace $\mathcal{D}\left(\pi_{\omega}\right) \subset \mathcal{H}_{\pi_{\omega}}$, a closed *-representation $\pi_{\omega}: \mathfrak{A} \rightarrow \mathcal{L}^{\dagger}\left(\mathcal{D}\left(\pi_{\omega}\right)\right)$ such that $\omega\left(b^{*} x a\right)=\left\langle\pi_{\omega}(x) \lambda_{\omega}(a) \mid \lambda_{\omega}(b)\right\rangle, \forall a, b, x \in \mathfrak{A}$. In this case, properties (L.1)(L.3) are automatically satisfied by $\omega$. Moreover, if $\mathfrak{A}$ possesses the unit, then $\omega$ is cyclically ${ }^{*}$-representable, i.e. there exists a vector $\xi_{\omega} \in \mathcal{D}\left(\pi_{\omega}\right)$ such that $\omega(x)=\left\langle\pi_{\omega}(x) \xi_{\omega} \mid \xi_{\omega}\right\rangle, \forall x \in \mathfrak{A}$ and $\xi_{\omega}$ is strongly cyclic. The representation $\pi_{\omega}$ is unique up to unitary equivalence.

Definition 2.1. Let $\left(\mathfrak{A}, \mathfrak{A}_{0}\right)$ be a quasi *-algebra without unit, its unitization is the couple $\left(\mathfrak{A}^{e}, \mathfrak{A}_{0}^{e}\right)$ where $\mathfrak{A}^{e}=\mathfrak{A} \oplus \mathbb{C}$ and $\mathfrak{A}_{0}^{e}=\mathfrak{A}_{0} \oplus \mathbb{C}$. The partial multiplication of $\mathfrak{A}$ is extended to $\mathfrak{A}^{e}$ as follows

$$
(x, \lambda) \cdot(a, \mu)=(x a+\mu x+\lambda a, \lambda \mu), \quad x \in \mathfrak{A}, a \in \mathfrak{A}_{0}, \lambda, \mu \in \mathbb{C}
$$

and the adjoint of an element $(x, \eta)$ is

$$
(x, \eta)^{*}=\left(x^{*}, \bar{\eta}\right), \quad x \in \mathfrak{A}, \eta \in \mathbb{C} .
$$

Together with the usual operations of the direct sum space, the previous partial multiplication and the involution make $\left(\mathfrak{A}^{e}, \mathfrak{A}_{0}^{e}\right)$ into a quasi ${ }^{*}$-algebra with unit $(0,1) \in \mathfrak{A}_{0}^{e}$.

Definition 2.2. A linear functional $\omega$, defined on a quasi ${ }^{*}$-algebra $\left(\mathfrak{A}, \mathfrak{A}_{0}\right)$ without unit is called extensible if $\omega$ is equal to the restriction to 
$\left(\mathfrak{A} \oplus\{0\}, \mathfrak{A}_{0} \oplus\{0\}\right)$ of some representable linear functional $\omega^{e}$ defined on the unitization $\left(\mathfrak{A}^{e}, \mathfrak{A}_{0}^{e}\right)$.

Before going forth, let us show that, in general, a representable positive linear functional $\omega$ defined on a quasi*-algebra $\left(\mathfrak{A}, \mathfrak{A}_{0}\right)$ without unit is not automatically extensible. In some cases it is not even possible to extend its restriction $\omega \uparrow \mathfrak{A}_{0}$ to the unitization of the ${ }^{*}$-algebra $\mathfrak{A}_{0}$, as the in following example.

Example 2.3. Let $\left(\mathfrak{A}, \mathfrak{A}_{0}\right)=\left(L^{1}(\mathbb{R}) \bigcap L^{2}(\mathbb{R}), C_{0}(\mathbb{R})\right)$. It is a quasi *algebra without unit. Let us consider the functional

$$
\omega(f)=\int_{\mathbb{R}} f(x) d x, \quad f \in L^{1}(\mathbb{R}) \bigcap L^{2}(\mathbb{R}) .
$$

It is easy to see that $\omega$ enjoys properties (L.1)-(L.3). Indeed, as for (L.3), $\forall f \in L^{1}(\mathbb{R}) \bigcap L^{2}(\mathbb{R})$ and $\forall g \in C_{0}(\mathbb{R})$,

$$
\left|\omega\left(f^{*} g\right)\right|=\left|\int_{\mathbb{R}} \overline{f(x)} g(x) d x\right| \leq\|f\|_{2}\|g\|_{2}=\gamma_{f} \omega\left(g^{*} g\right)^{1 / 2}
$$

Hence $\omega$ is representable on $L^{1}(\mathbb{R}) \bigcap L^{2}(\mathbb{R})$.

For $\omega$ to be extensible to the unitization $\left(L^{1}(\mathbb{R}) \cap L^{2}(\mathbb{R})\right) \oplus \mathbb{C}$ it is necessary that it is extensible to the unitization of $C_{0}(\mathbb{R})$, but this does not happen; indeed, it is not Hilbert bounded; were it so, one should have

$$
\|\omega\|_{H}=\sup \left\{|\omega(g)|^{2} ; g \in C_{0}(\mathbb{R}), \omega\left(g^{*} g\right)=1\right\}<\infty,
$$

but if we take the functions $f_{n}(x) \in C_{0}(\mathbb{R})$ with

$$
f_{n}(x)= \begin{cases}n x+n^{2}+1 & \text { if } x \in[-n-1 / n,-n[ \\ 1 & \text { if } x \in[-n, n] \\ -n x+n^{2}+1, & \text { if } x \in] n, n+1 / n] \\ 0 & \text { elsewhere, }\end{cases}
$$

we get $\omega\left(f_{n}\right)=\int_{\mathbb{R}} f_{n}(x) d x \geq 2 n,\left|\omega\left(f_{n}\right)\right|^{2}=\left|\int_{\mathbb{R}} f_{n}(x) d x\right| \geq 4 n^{2}$ and $\omega\left(f_{n}^{*} f_{n}\right)=\int_{\mathbb{R}}\left|f_{n}(x)\right|^{2} d x \leq \int_{-2 n}^{2 n} d x=4 n$, hence the quotient $\frac{|\omega(g)|^{2}}{\omega\left(g^{*} g\right)}>n$. It is thus clear that we cannot extend $\omega$ to the unitized quasi ${ }^{*}$-algebra.

We will maintain the notation of [11].

\section{Representable extensions to a unitized quasi *-algebra}

Let $\left(\mathfrak{A}, \mathfrak{A}_{0}\right)$ be a quasi *-algebra without unit and $\omega$ be a positive linear functional defined on $\left(\mathfrak{A}, \mathfrak{A}_{0}\right)$. Let $\omega$ be hermitian (i.e., $\omega\left(x^{*}\right)=\overline{\omega(x)}$, $\forall x \in \mathfrak{A}$ ), then (L.2) is automatically satisfied. Assume, in addition, that 
$\omega_{0}=\omega\left\lceil\mathfrak{A}_{0}\right.$ is also Hilbert bounded on the ${ }^{*}$-algebra $\mathfrak{A}_{0}$; i.e., the following property holds

(HB) there exists $\delta>0$ such that

$$
\left|\omega_{0}(a)\right|^{2} \leq \delta \omega_{0}\left(a^{*} a\right), \quad \forall a \in \mathfrak{A}_{0} .
$$

Then $\omega_{0}$ is positive (thus property (L.1) is satisfied). The Hilbert bound $\left\|\omega_{0}\right\|_{H}$ is defined by

$$
\left\|\omega_{0}\right\|_{H}=\sup \left\{\left|\omega_{0}(a)\right|^{2} ; a \in \mathfrak{A}_{0}, \omega_{0}\left(a^{*} a\right) \leq 1\right\} ;
$$

if $\mathfrak{A}_{0}$ has a unit $e$, then as a simple consequence of the Cauchy-Schwarz inequality, $\left|\omega_{0}(a)\right|^{2} \leq \omega_{0}(e) \omega_{0}\left(a^{*} a\right), \forall a \in \mathfrak{A}_{0}$; i.e. $\omega_{0}$ is Hilbert bounded and $\left\|\omega_{0}\right\|_{H}=\omega_{0}(e)$.

If (HB) holds true, then it is known that $\omega_{0}$ can be extended to $\mathfrak{A}_{0}^{e}$; indeed, the functional $\omega_{0}^{e}$ defined on the unitized *-algebra $\mathfrak{A}_{0}^{e}$ by

$$
\omega_{0}^{e}((a, \lambda))=\omega_{0}(a)+\lambda \gamma_{0}, \quad(a, \lambda) \in \mathfrak{A}_{0}^{e}
$$

with $\gamma_{0} \geq\left\|\omega_{0}\right\|_{H}$ is positive. If $\xi$ is the cyclic vector of the GNS representation of $\omega_{0}^{e}$, then

$$
\left|\omega_{0}(a)\right|^{2} \leq\|\xi\|^{2} \omega_{0}\left(a^{*} a\right), \quad \forall a \in \mathfrak{A}_{0}
$$

with $\|\xi\|^{2}=\left\|\omega_{0}^{e}\right\|_{H}=\omega_{0}^{e}((0,1))=\gamma_{0} \geq\left\|\omega_{0}\right\|_{H}$, then (11) can be rewritten as

$$
\omega_{0}^{e}((a, \lambda))=\omega_{0}(a)+\lambda\|\xi\|^{2}, \quad(a, \lambda) \in \mathfrak{A}_{0}^{e} .
$$

Remark 3.1. If $\mathfrak{A}_{0}$ is a $C^{*}$-algebra without unit and $\vartheta$ is a positive functional on $\mathfrak{A}_{0}$, then $\vartheta$ is automatically Hilbert bounded and therefore extensible to $\mathfrak{A}_{0}^{e}$ (see, e.g. [5, Corollary 2.3.13]).

Assume now that $\omega$ satisfies property (L.3) too. Hence it is representable. Now we extend $\omega$ to $\mathfrak{A}^{e}$, for every $(x, \eta) \in \mathfrak{A}^{e}$ by defining 1

$$
\omega^{e}((x, \eta)):=\omega(x)+\eta \gamma
$$

with $\gamma \geq\|\xi\|^{2}$.

We want to present some conditions for the representability of $\omega^{e}$. Condition (L.1) holds also for $\omega^{e}$ on $\mathfrak{A}_{0}^{e}$; indeed it suffices to observe that

\footnotetext{
${ }^{1}$ In order to lighten the notation we do not stress the dependence of $\omega^{e}$ on $\gamma$; to be more precise to each $\gamma \geq\|\xi\|^{2}$ is associated a different extension of $\omega$.
} 
$\forall(a, \lambda) \in \mathfrak{A}_{0}^{e}$, by using (2) and hermiticity

$$
\begin{aligned}
\omega^{e}\left(\left(a^{*}, \bar{\lambda}\right) \cdot(a, \lambda)\right) & =\omega\left(a^{*} a\right)+\lambda \omega\left(a^{*}\right)+\bar{\lambda} \omega(a)+|\lambda|^{2} \gamma \\
& \geq \omega\left(a^{*} a\right)-2|\lambda||\omega(a)|+|\lambda|^{2} \gamma \\
& \geq \omega\left(a^{*} a\right)-2|\lambda| \gamma^{1 / 2} \omega\left(a^{*} a\right)^{1 / 2}+|\lambda|^{2} \gamma \\
& =\left(\omega\left(a^{*} a\right)^{1 / 2}-|\lambda| \gamma^{1 / 2}\right)^{2} \geq 0 .
\end{aligned}
$$

Now, the hermiticity of $\omega$ implies that $\omega^{e}$ is hermitian too. Indeed,

$$
\omega^{e}\left(\left(x^{*}, \bar{\eta}\right)\right)=\omega\left(x^{*}\right)+\bar{\eta} \gamma=\overline{\omega(x)+\eta \gamma}=\overline{\omega^{e}((x, \eta))}, \quad \forall(x, \eta) \in \mathfrak{A}^{e} .
$$

As for property (L.3), even if $\omega$ is representable, in general it is not true for $\omega^{e}$, as the following example shows.

Example 3.2. Let us consider the quasi ${ }^{*}$-algebra $\left(\mathfrak{A}, \mathfrak{A}_{0}\right)$, where $\mathfrak{A}=\ell_{2}$ is, as usual, the space of square summable complex sequences and $\mathfrak{A}_{0}$ the *-algebra of all finite sequences, i.e.:

$$
\mathfrak{A}_{0}:=\left\{\boldsymbol{a}=\left(a_{n}\right): \exists N \in \mathbb{N}, a_{n}=0, \forall n>N\right\},
$$

with componentwise multiplication, and let us define a linear functional $\omega$ on $\ell_{2}$ by

$$
\omega\left(\left(x_{n}\right)\right)=\sum_{n=1}^{+\infty} x_{n}, \quad\left(x_{n}\right) \in \ell_{2} .
$$

As we showed in [4, $\omega$ is representable both as functional defined on the whole quasi *-algebra and if we restrict it on the *-algebra $\mathfrak{A}_{0}$. However, its extension defined as in (3), does not enjoy property (L.3). Indeed, if we consider $\boldsymbol{x}=\left(x_{n}\right)$ with $x_{n}=1 / n, \forall n \in \mathbb{N}$, we have, for $\mu \neq 0$ and for all $a \in \mathfrak{A}_{0}$

$\left|\omega^{e}\left((\boldsymbol{x}, \eta)^{*} \cdot(\boldsymbol{a}, \mu)\right)\right|^{2}=\left|\omega\left(\boldsymbol{x}^{*} \boldsymbol{a}\right)+\mu \omega\left(\boldsymbol{x}^{*}\right)+\bar{\eta} \omega(\boldsymbol{a})+\bar{\eta} \mu \gamma\right|^{2}=\left|K+\mu \sum_{n=1}^{+\infty} 1 / n\right|^{2}=\infty$ since $K=\omega\left(\boldsymbol{x}^{*} \boldsymbol{a}\right)+\bar{\eta} \omega(\boldsymbol{a})+\bar{\eta} \mu \gamma$ contains only finite sums.

It is then necessary to introduce a further condition in order that (L.3) holds for $\omega^{e}$. To do this, let us define

$$
p_{x}(\omega):=\sup \left\{\left|\omega\left(x^{*} a\right)\right|^{2}: a \in \mathfrak{A}_{0}, \omega\left(a^{*} a\right)=1\right\} .
$$

The functional $x \mapsto p_{x}(\omega)^{1 / 2}$ is a seminorm: indeed,

i) $p_{x}(\omega) \geq 0 \forall x \in \mathfrak{A}$, then $p_{x}(\omega)^{1 / 2} \geq 0$,

ii) if $x=0$, then $p_{x}(\omega)^{1 / 2}=0$, 
iii) $p_{\lambda x}(\omega)^{1 / 2}=|\lambda| p_{x}(\omega)^{1 / 2} \forall x \in \mathfrak{A}, \lambda \in \mathbb{C}$,

iv) $p_{x+y}(\omega)^{1 / 2} \leq p_{x}(\omega)^{1 / 2}+p_{y}(\omega)^{1 / 2}$.

Now we are ready to introduce a necessary and sufficient condition for $\omega$ to be extensible:

(EHB) there exists $\zeta>0$ such that

$$
\left|\omega\left(x^{*}\right)\right| \leq \zeta p_{x}(\omega)^{1 / 2}, \quad \forall x \in \mathfrak{A} .
$$

Remark 3.3. If $\left(\mathfrak{B}, \mathfrak{B}_{0}\right)$ is a quasi *algebra with unit $e \in \mathfrak{B}_{0}$, then (EHB) is automatically fulfilled by a positive linear functional $\vartheta$ defined on it; indeed, for every $x \in \mathfrak{B}$

$$
\left|\vartheta\left(x^{*}\right)\right|^{2}=\left|\vartheta\left(x^{*} e\right)\right|^{2} \leq \sup \left\{\left|\vartheta\left(x^{*} a\right)\right|^{2}: a \in \mathfrak{B}_{0}, \vartheta\left(a^{*} a\right)=1\right\}=p_{x}(\vartheta)^{1 / 2} .
$$

Hence condition (EHB) is necessary for the extensibility of a positive linear functional $\omega$ defined on a quasi *algebra without unit, to the unitized quasi *-algebra; moreover, (EHB) is also a sufficient condition for the extensibility of $\omega$ as Proposition 3.5 below shows.

Condition (EHB) is a natural one; indeed, the following lemma holds.

Lemma 3.4. Let us consider a linear functional $\omega$ on a quasi *algebra $\left(\mathfrak{A}, \mathfrak{A}_{0}\right)$ and suppose that it enjoys property (L.1). If $\omega$ enjoys (EHB), then it enjoys property $(H B)$ too. The two conditions are equivalent on $\mathfrak{A}_{0}$.

Proof. It suffices to prove that properties (HB) and (EHB) coincide on $\mathfrak{A}_{0}$. Let $b \in \mathfrak{A}_{0}$, then $p_{b}(\omega)=\sup \left\{\left|\omega\left(b^{*} a\right)\right|^{2} ; \omega \in \mathfrak{A}_{0} \omega\left(a^{*} a\right)=1\right\}$; by the Cauchy-Schwarz inequality $\left|\omega\left(b^{*} a\right)\right|^{2} \leq \omega\left(b^{*} b\right) \omega\left(a^{*} a\right)$ we get that $p_{b}(\omega) \leq$ $\omega\left(b^{*} b\right)$. More precisely, the equality holds: indeed, if $\omega\left(b^{*} b\right)=0$ then $p_{b}(\omega)=0$; other-wise take $a=b / \omega\left(b^{*} b\right)^{1 / 2} \in \mathfrak{A}_{0}$; then, $\omega\left(a^{*} a\right)=1$ and $p_{b}(\omega)=\omega\left(b^{*} b\right)$.

According the previous lemma, (EHB) extends (HB) to the whole quasi *-algebra, whence the acronym (EHB).

The main result of this paper then holds.

Proposition 3.5. Let $\omega$ be a hermitian linear functional defined on a quasi *-algebra $\left(\mathfrak{A}, \mathfrak{A}_{0}\right)$ without unit, such that property (L.3) holds. If (EHB) holds too, then the extension $\omega^{e}$ to the unitized quasi ${ }^{*}$-algebra $\left(\mathfrak{A}^{e}, \mathfrak{A}_{0}^{e}\right)$ is cyclically representable, i.e. the functional $\omega$ is extensible to the unitized quasi *-algebra. 
Proof. Let us consider the functional $\omega^{e}$ defined in (3). Property (HB) holds for $\omega_{0}=\omega\left\lceil\mathfrak{A}_{0}\right.$ by Lemma 3.4. We have already observed that properties (L.1) and (L.2) hold for $\omega^{e}$, because of (HB) and the hermiticity of $\omega$. It remains to verify that, if (L.3) and (EHB) hold for $\omega$, property (L.3) holds for $\omega^{e}$ too. Let us estimate the squared modulus of $\omega^{e}\left(\left(x^{*}, \bar{\eta}\right) \cdot(a, \mu)\right)$

$$
\begin{aligned}
\mid \omega^{e} & \left.\left(\left(x^{*}, \bar{\eta}\right) \cdot(a, \mu)\right)\right|^{2}= \\
= & \left|\omega\left(x^{*} a\right)\right|^{2}+|\eta|^{2}|\omega(a)|^{2}+|\mu|^{2}\left|\omega\left(x^{*}\right)\right|^{2}+|\eta|^{2}|\mu|^{2} \gamma^{2}+2 \Re\left(|\eta|^{2} \bar{\mu} \gamma \omega(a)\right)+ \\
& +2 \Re\left(\left[\omega\left(x^{*}\right)+\bar{\eta} \gamma\right] \mu \overline{\omega\left(x^{*} a\right)}+\bar{\eta} \omega(a) \overline{\omega\left(x^{*} a\right)}+\overline{\eta \mu} \omega(x) \omega(a)+\eta|\mu|^{2} \gamma \omega\left(x^{*}\right)\right) \\
\leq & \left(\gamma_{x}^{2}+|\eta|^{2} \gamma\right) \omega\left(a^{*} a\right)+\left(\frac{\zeta^{2} p_{x}(\omega)}{\gamma}+|\eta|^{2} \gamma\right)|\mu|^{2} \gamma+2|\eta|^{2} \gamma \Re(\bar{\mu} \omega(a))+ \\
& +2\left[\left(\zeta p_{x}(\omega)^{1 / 2}+|\eta| \gamma\right)|\mu| \gamma_{x} \omega\left(a^{*} a\right)^{1 / 2}+|\eta| \gamma^{1 / 2} \gamma_{x} \omega\left(a^{*} a\right)+\right. \\
& \left.+\left(\gamma^{1 / 2} \omega\left(a^{*} a\right)^{1 / 2}+|\mu| \gamma\right)|\eta||\mu| \zeta p_{x}(\omega)^{1 / 2}\right] \\
\leq & \left(\gamma_{x}+|\eta| \gamma^{1 / 2}\right)^{2} \omega\left(a^{*} a\right)+\left(\frac{\zeta p_{x}(\omega)^{1 / 2}}{\gamma^{1 / 2}}+|\eta| \gamma^{1 / 2}\right)^{2}|\mu|^{2} \gamma+2|\eta|^{2} \gamma \Re(\bar{\mu} \omega(a)) \\
& +2\left[\left(\zeta p_{x}(\omega)^{1 / 2}+|\eta| \gamma\right) \gamma_{x}+\gamma^{1 / 2}|\eta| \zeta p_{x}(\omega)^{1 / 2}+|\eta|^{2} \gamma^{3 / 2}\right]|\mu| \omega\left(a^{*} a\right)^{1 / 2} \\
\leq & {\left[\left(\gamma_{x}+|\eta| \gamma^{1 / 2}\right) \omega\left(a^{*} a\right)^{1 / 2}+\left(\frac{\zeta p_{x}(\omega)^{1 / 2}}{\gamma^{1 / 2}}+|\eta| \gamma^{1 / 2}\right)|\mu| \gamma^{1 / 2}\right]^{2} } \\
& +2|\eta|^{2} \gamma \Re(\bar{\mu} \omega(a)) .
\end{aligned}
$$

Put

$$
K_{(x, \eta)}:=\max \left\{\gamma_{x}+|\eta| \gamma^{1 / 2}, \frac{\zeta p_{x}(\omega)^{1 / 2}}{\gamma^{1 / 2}}+|\eta| \gamma^{1 / 2}\right\}
$$

and

$$
\Gamma_{(x, \eta)}:=\max \left\{2\left(K_{(x, \eta)}\right)^{2},|\eta|^{2} \gamma\right\}
$$

Then

$$
\begin{aligned}
& \left|\omega^{e}\left(\left(x^{*}, \bar{\eta}\right) \cdot(a, \mu)\right)\right| \leq\left(K_{(x, \eta)}\right)^{2}\left[\omega\left(a^{*} a\right)^{1 / 2}+|\mu| \gamma^{1 / 2}\right]^{2}+2|\eta|^{2} \gamma \Re(\bar{\mu} \omega(a)) \\
& \leq 2\left(K_{(x, \eta)}\right)^{2}\left[\omega\left(a^{*} a\right)+|\mu|^{2} \gamma\right]+2|\eta|^{2} \gamma \Re(\bar{\mu} \omega(a)) \\
& \leq \Gamma_{(x, \eta)}\left[\omega\left(a^{*} a\right)+|\mu|^{2} \gamma+2 \Re(\bar{\mu} \omega(a))\right]=\Gamma_{(x, \eta)} \omega^{e}\left(\left(a^{*}, \bar{\mu}\right) \cdot(a, \mu)\right) .
\end{aligned}
$$

The functional $\omega^{e}$ is then representable on the whole $\left(\mathfrak{A}^{e}, \mathfrak{A}_{0}^{e}\right)$. 
Corollary 3.6. Let $\omega$ be a hermitian linear functional defined on a quasi *-algebra $\left(\mathfrak{A}, \mathfrak{A}_{0}\right)$ without unit, such that property (EHB) holds. Then the representability of $\omega$ is equivalent to its extensibility.

Example 3.7. A very simple example of the situation described in Proposition 3.5 is obtained by considering the quasi *algebra $\left(\mathfrak{A}, C_{0}(\mathbb{R})\right)$, with $\mathfrak{A}=\left\{f \in C(\mathbb{R}): \int_{\mathbb{R}}|f(x)| e^{-x} d x<\infty\right\}, C_{0}(\mathbb{R})$ the ${ }^{*}$-algebra of continuous functions with compact support and the linear functional defined on it $\omega(f)=f(0)$. It is easy to prove that $\omega$ is hermitian and that (L.1) and (L.3) hold. The functional $\omega$ enjoys also property (EHB) on $\mathfrak{A}$. Indeed,

$$
\begin{aligned}
p_{x}(\omega) & =\sup \left\{\left|\omega\left(f^{*} g\right)\right|^{2}: g \in \mathfrak{A}_{0}, \omega\left(g^{*} g\right)=1\right\} \\
& =\sup \left\{|\overline{f(0)} g(0)|^{2}: g \in \mathfrak{A}_{0},|g(0)|^{2}=1\right\}=|\overline{f(0)}|^{2} .
\end{aligned}
$$

It is then possible to extend $\omega$ to a positive linear functional on the unitization of $\left(\mathfrak{A}, \mathfrak{A}_{0}\right): \omega^{e}((f, \eta)):=\omega(f)+\eta \gamma=f(0)+\eta \gamma$ with $\gamma \geq\|\xi\|^{2} \geq$ $\left\|\omega_{0}\right\|_{H}=1$.

We are now able to extend a well-known theorem for positive linear functionals on *-algebras without unit, to positive linear functionals defined on quasi *-algebras without unit, by summarizing the remarks and results given until now.

Theorem 3.8. Let $\omega$ be a linear functional on $\left(\mathfrak{A}, \mathfrak{A}_{0}\right)$ for which (L.1) holds and consider the following statements.

(i) $\omega^{e}: \mathfrak{A}^{e} \rightarrow \mathbb{C}$ defined by

$$
\omega^{e}((x, \lambda))=\omega(x)+\gamma \lambda, \quad(x, \lambda) \in \mathfrak{A}^{e}
$$

is a representable linear functional on $\mathfrak{A}^{e}$, for every $\gamma \geq\left\|\omega_{0}^{e}\right\|_{H}$;

(ii) there exists a ${ }^{*}$-representation $\pi$ of $\mathfrak{A}$ and a vector $\xi \in \mathcal{D}\left(\pi_{\omega^{e}}\right)$ such that $\omega(x)=\langle\pi(x) \xi \mid \xi\rangle, \forall x \in \mathfrak{A} ;$

(iii) $\omega$ is hermitian, enjoys (HB) and for every $x \in \mathfrak{A}$ there exists a vector $\zeta_{\omega}(x) \in \mathcal{H}_{\omega}$ such that

$$
\omega\left(x^{*} a\right)=\left\langle\lambda_{\omega}(a) \mid \zeta_{\omega}(x)\right\rangle, \quad \forall a \in \mathfrak{A}_{0} .
$$

Then the following implications hold

$$
(i) \Rightarrow(i i) \Rightarrow(i i i)
$$

\footnotetext{
${ }^{2}$ In general, it is not a cyclic vector for $\pi$, but only for $\pi_{\omega^{e}}$ a ${ }^{*}$-representation of $\omega^{e}$.
} 
Moreover, if there exists $\zeta>0$ such that

$$
\left|\omega\left(x^{*}\right)\right| \leq \zeta p_{x}(\omega)^{1 / 2}, \quad \forall x \in \mathfrak{A}
$$

then the three statements $(i),($ ii) and (iii) are equivalent and also equivalent to the following ones

(iv) $\omega$ is extensible;

(v) $\omega$ is hermitian and enjoys property (L.3);

(vi) $\omega$ is hermitian and representable.

Proof. $(i) \Rightarrow($ ii $)$ : it suffices to consider the GNS representation of the unitized quasi *-algebra $\left(\mathfrak{A}^{e}, \mathfrak{A}_{0}^{e}\right)$ constructed (as in [1]) from $\omega^{e}$, restrict it to $\left(\mathfrak{A} \oplus\{0\}, \mathfrak{A}_{0} \oplus\{0\}\right)$, define $\pi(x):=\pi_{\omega^{e}}(x, 0), \forall x \in \mathfrak{A}$ and take $\xi=\xi_{\omega^{e}}=\lambda_{\omega^{e}}((0,1)) \in \mathcal{D}\left(\pi_{\omega^{e}}\right)$ (but, in general, $\left.\xi \notin \mathcal{D}\left(\pi_{\omega^{e}} \uparrow \mathfrak{A} \oplus\{0\}\right)\right)$.

$($ ii $) \Rightarrow($ iii $)$ : if $\pi$ is a ${ }^{*}$-representation as described in $(i i)$, then $\omega$ is hermitian, and $\forall a \in \mathfrak{A}_{0}$

$$
|\omega(a)|^{2}=|\langle\pi(a) \xi \mid \xi\rangle|^{2} \leq\|\xi\|^{2}\|\pi(a) \xi\|^{2}=\|\xi\|^{2}\left\langle\pi\left(a^{*} a\right) \xi \mid \xi\right\rangle=\|\xi\|^{2} \omega\left(a^{*} a\right)
$$

further $\forall x \in \mathfrak{A}$

$$
\omega\left(x^{*} a\right)=\left\langle\pi\left(x^{*} a\right) \xi \mid \xi\right\rangle=\langle\pi(a) \xi \mid \pi(x) \xi\rangle=\left\langle\lambda_{\omega}(a) \mid \zeta_{\omega}(x)\right\rangle, \quad \forall a \in \mathfrak{A}_{0}
$$

remembering that $\xi=\lambda_{\omega_{0}^{e}}((0,1))$.

Let from now on (EHB) hold for $\omega$.

$($ iii $) \Rightarrow(i v)$ : it follows easily that (L.3) holds for $\omega$. Then, by Proposition 3.5 we get the result.

$(i v) \Rightarrow(v)$ : assume that $\omega^{\prime}$ is a representable linear functional which extends $\omega$ to $\mathfrak{A}^{e}$. Then, $\omega$ is hermitian and (L.3) holds for $\omega$ too.

$(v) \Rightarrow(i)$ : by hypotheses $\left\|\omega_{0}^{e}\right\|_{H}<\infty$ and let $\gamma \geq\left\|\omega_{0}^{e}\right\|_{H}$. The proof of (L.1) for $\omega^{e}$ is standard, (L.2) follows from hermiticity of $\omega$ and (L.3) for $\omega^{e}$ follows from Proposition 3.5 .

$(i v) \Rightarrow(v i)$ : see Corollary 3.6

Remark 3.9. Let $(H B)$ hold for $\omega_{0}=\omega\left\lceil\mathfrak{A}_{0}\right.$. Then, the linear functional

$$
F\left(\lambda_{\omega_{0}}(a)\right)=\omega_{0}(a)
$$

is Riesz representable, i.e. there exists $\xi_{\omega_{0}} \in \lambda_{\omega_{0}}\left(\mathfrak{A}_{0}\right)$ such that

$$
\omega_{0}(a)=\left\langle\lambda_{\omega_{0}}(a) \mid \xi_{\omega_{0}}\right\rangle .
$$

Now, $\forall b \in \mathfrak{A}_{0}$,

$$
\omega_{0}(b a)=\left\langle\lambda_{\omega_{0}}(b a) \mid \xi_{\omega_{0}}\right\rangle=\left\langle\pi_{\omega_{0}}(b) \lambda_{\omega_{0}}(a) \mid \xi_{\omega_{0}}\right\rangle
$$


and, on the other hand

$$
\omega_{0}(b a)=\left\langle\lambda_{\omega_{0}}(a) \mid \lambda_{\omega_{0}}\left(b^{*}\right)\right\rangle,
$$

hence $\pi_{\omega_{0}}(b)^{*} \xi_{\omega_{0}}=\lambda_{\omega_{0}}\left(b^{*}\right)$; we can conclude that $\xi_{\omega_{0}} \in \mathcal{D}\left(\pi_{\omega_{0}}^{*}\right)$.

If $\pi_{\omega_{0}}$ is self-adjoint, then $\lambda_{\omega_{0}}(a)=\pi_{\omega_{0}}(a) \xi_{\omega_{0}}, a \in \mathfrak{A}_{0}$. In particular, if $\pi_{\omega_{0}}$ is bounded (i.e., if $\omega_{0}$ is admissible), then $\xi_{\omega_{0}}$ is a cyclic vector for $\pi_{\omega_{0}}$ and $\omega_{0}(a)=\left\langle\pi_{\omega_{0}}(a) \xi_{\omega_{0}} \mid \xi_{\omega_{0}}\right\rangle$; hence $\omega_{0}$ is strongly cyclically representable. Conversely, with the same argument as in $(i i) \Rightarrow($ iii $)$ in Theorem 3.8 it is clear that, if $\omega$ is cyclically representable, then it enjoys (HB). We cannot conclude it from the Theorem 3.8 directly, because the representation in (ii) is not cyclic, in the sense that the vector $\xi$ is not cyclic for $\pi$.

There are cases in which property (EHB) does not hold, as the following examples show.

Example 3.10. Let us consider both the quasi *algebra and the linear functional of Example 3.2. There, we already observed that $\omega$ is representable as a functional defined on the whole quasi *algebra (hence (L.3) is satisfied see also [4]) as well as restriction to the ${ }^{*}$-algebra $\mathfrak{A}_{0}$. The functional $\omega$ is hermitian, but it does not enjoy property (EHB).

Let us calculate, for any $\boldsymbol{a} \in \mathfrak{A}_{0}$ and for a fixed $\boldsymbol{x} \in \ell_{2}$,

$$
\frac{\left|\omega\left(\boldsymbol{x}^{*} \boldsymbol{a}\right)\right|^{2}}{\omega\left(\boldsymbol{a}^{*} \boldsymbol{a}\right)}=\frac{\left|\sum_{n=1}^{N} \overline{x_{n}} a_{n}\right|^{2}}{\sum_{n=1}^{N}\left|a_{n}\right|^{2}} \leq \frac{\sum_{n=1}^{N}\left|x_{n}\right|^{2} \sum_{n=1}^{N}\left|a_{n}\right|^{2}}{\sum_{n=1}^{N}\left|a_{n}\right|^{2}}=\sum_{n=1}^{N}\left|x_{n}\right|^{2}<\infty .
$$

Hence $p_{x}(\omega) \leq\|\boldsymbol{x}\|_{2}^{2}$, but property (EHB) does not hold. Indeed, it suffices to consider a sequence such that $\left|\omega\left(\boldsymbol{x}^{*}\right)\right|>\zeta\|\boldsymbol{x}\|_{2} \geq \zeta p_{x}(\omega)^{1 / 2}$, for every $\zeta>0$ i.e. such that

$$
\left|\sum_{n=1}^{\infty} \overline{x_{n}}\right|=\left|\sum_{n=1}^{\infty} x_{n}\right|>\zeta\left(\sum_{n=1}^{\infty}\left|x_{n}\right|^{2}\right)^{1 / 2}, \quad \forall \zeta>0 .
$$

An easy example of this situation is obtained by taking $\boldsymbol{x}=\left(\frac{1}{n}\right)_{n \in \mathbb{N}^{N}}$. Hence, the task of extending $\omega$ to the unitization of $\ell_{2}$ is hopeless, here.

Example 3.11. Let us consider the same functional and the quasi *algebra without unit of Example 2.3. Let us consider the functions $f_{n} \in L^{1}(\mathbb{R}) \cap L^{2}(\mathbb{R})$ defined as

$$
f_{n}(x)= \begin{cases}1 / \sqrt{2 n}, & x \in[-n, n] \\ 0, & \text { otherwise }\end{cases}
$$


They have unit norm, but

$$
\left|\omega\left(f_{n}^{*}\right)\right|=\left|\int_{-n}^{n} 1 / \sqrt{2 n} d x\right|=\frac{1}{\sqrt{2 n}} 2 n=\sqrt{2 n} \rightarrow \infty, \text { as } n \rightarrow \infty .
$$

Then, property (EHB) does not hold. We already noted in Example 2.3 that this functional is not extensible.

If $\omega$ is hermitian and properties (HB) and (L.3) hold but condition (EHB) is not fulfilled on the whole $\mathfrak{A}$, it is possible to extend $\omega$ to a quasi *-algebra with unit $\left(\mathcal{D}\left(\omega^{e}\right), \mathfrak{A}_{0}^{e}\right)$ with $\mathcal{D}\left(\omega^{e}\right) \subset \mathfrak{A}^{e}$. For this, let us restrict the linear functional $\omega^{e}$ defined in (3) to the vector space

$$
\begin{aligned}
\mathcal{D}\left(\omega^{e}:\right. & \models\left\{(x, \eta) \in \mathfrak{A}^{e}: \exists \delta_{(x, \eta)}>0\right. \text { s.t. } \\
& \left.\left|\omega^{e}\left((x, \eta)^{*} \cdot(a, \mu)\right)\right| \leq \delta_{(x, \eta)} \omega^{e}\left((a, \mu)^{*} \cdot(a, \mu)\right)^{1 / 2}, \forall(a, \mu) \in \mathfrak{A}_{0}^{e}\right\} .
\end{aligned}
$$

We notice that $\mathcal{D}\left(\omega^{e}\right)$ contains $\mathfrak{A}_{0}^{e}$ and $\omega_{0}^{e}$ satisfies $(\mathrm{HB})$ since $\omega_{0}^{e}=\omega^{e} \uparrow \mathfrak{A}_{0}^{e}$ is a positive linear functional on a ${ }^{*}$-algebra (with unit) and hence it satisfies the Cauchy-Schwarz inequality. As for the algebraic structure of $\mathcal{D}\left(\omega^{e}\right)$, it is in general a nonempty subspace of $\mathfrak{A}^{e}$. But there are situations where something more can be said. For instance, let us assume that the following condition of admissibility holds:

$(\mathrm{ADM}) \forall(b, \nu) \in \mathfrak{A}_{0}^{e}$ there exists $\delta_{(b, \nu)}>0$ such that

$$
\omega_{0}^{e}\left((a, \mu)^{*} \cdot(b, \nu)^{*} \cdot(b, \nu) \cdot(a, \mu)\right) \leq \delta_{(b, \nu)} \omega_{0}^{e}\left((a, \mu)^{*} \cdot(a, \mu)\right), \forall(a, \mu) \in \mathfrak{A}_{0}^{e} .
$$

Remark 3.12. If $\mathfrak{A}_{0}$ is a $C^{*}$-algebra, then $(A D M)$ is automatically satisfied.

Let us suppose that $\mathcal{D}\left(\omega^{e}\right)$ is stable under involution.

Proposition 3.13. Let $\omega$ be a hermitian linear functional on a quasi *algebra without unit, satisfying (HB) and (L.3). If $\omega_{0}^{e}$ satisfies (ADM) and $\mathcal{D}\left(\omega^{e}\right)$ is stable under involution, then $\left(\mathcal{D}\left(\omega^{e}\right), \mathfrak{A}_{0}^{e}\right)$ is a quasi ${ }^{*}$-algebra with unit.

Proof. The vector space $\mathcal{D}\left(\omega^{e}\right)$ contains $\mathfrak{A}_{0}^{e}$ and is ${ }^{*}$-stable. Besides, by $(\mathrm{ADM}),(b, \nu) \cdot(x, \eta) \in \mathcal{D}\left(\omega^{e}\right)$, with $(x, \eta) \in \mathcal{D}\left(\omega^{e}\right)$ and $(b, \nu) \in \mathfrak{A}_{0}^{e}$.

Properties (L.1)-(L.3) are hence satisfied by $\omega^{e}$ on $\mathcal{D}\left(\omega^{e}\right)$ and $\omega_{\mathcal{D}}^{e}:=\omega^{e} \uparrow$ $\mathcal{D}\left(\omega^{e}\right)$ is representable.

Let us maintain the notation of Section 2, for every $(x, \eta) \in \mathcal{D}\left(\omega^{e}\right)$, the

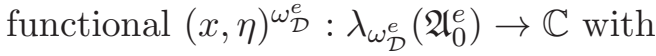

$$
(x, \eta)^{\omega_{\mathcal{D}}^{e}}\left(\lambda_{\omega_{\mathcal{D}}^{e}}(a, \mu)\right):=\omega_{\mathcal{D}}^{e}\left((x, \eta)^{*} \cdot(a, \mu)\right),
$$


is linear and bounded. By applying the Riesz lemma, we can prove the following.

Theorem 3.14. Let $\omega$ be a hermitian linear functional on a quasi *-algebra $\left(\mathfrak{A}, \mathfrak{A}_{0}\right)$ without unit, satisfying properties (HB) and (L.3). If $\left(\mathcal{D}\left(\omega^{e}\right), \mathfrak{A}_{0}^{e}\right)$ is a quasi ${ }^{*}$-algebra with unit then $\omega_{\mathcal{D}}^{e}$ is representable.

By Proposition 3.13, then we have the following

Corollary 3.15. Let $\omega$ be a hermitian linear functional on a quasi *algebra $\left(\mathfrak{A}, \mathfrak{A}_{0}\right)$ without unit, satisfying properties (HB) and (L.3). If $\omega_{0}^{e}$ satisfies also $(A D M)$ and $\mathcal{D}\left(\omega^{e}\right)$ is stable under involution, then $\omega_{\mathcal{D}}^{e}$ is representable on the quasi *-algebra with unit $\left(\mathcal{D}\left(\omega^{e}\right), \mathfrak{A}_{0}^{e}\right)$.

Remark 3.16. If $\omega_{0}^{e}$ satisfies (ADM), then the GNS-representation restricted to $\mathfrak{A}_{0}^{e}$, constructed from $\omega_{\mathcal{D}}^{e}$, is bounded.

Acknowledgment. The author wishes to thank Prof. A. Inoue and Prof. C. Trapani for their fruitful comments.

\section{REFERENCES}

[1] J-P. Antoine, A. Inoue, and C. Trapani, Partial *-Algebras and Their Operator Realizations, (Mathematics and Its Applications: Vol. 553). Kluwer Academic Publishers, 2002.

[2] J-P. Antoine, G. Bellomonte and C. Trapani, Fully representable and ${ }^{*}$-semisimple topological partial *algebras Studia Math., no. 2208 (2012), 167-194 DOI: 10.4064/sm208-2-4.

[3] F. Bagarello, C. Trapani and S. Triolo, Representable states on quasi-local quasi *algebras, J. Math. Phys., 52 (2011), no. 1, 013510, 11 pp.

[4] G. Bellomonte, Quasi *algebras arising from extensions of positive linear functionals. Rend. Circ. Mat. Palermo (2) Suppl. 82 (2010), 235-249.

[5] O. Bratteli, and D.W. Robinson, Operator Algebras and Quantum Statistical Mechanics, Vol. 1 (Texts and monographs in physics). Springer-Verlag 1979.

[6] M. Fragoulopoulou, C. Trapani and S. Triolo, Locally convex quasi *algebras with sufficiently many *-representations, J. Math. Anal. Appl. 388 (2012), 1180-1193.

[7] T.W. Palmer, Banach Algebras and the General Theory of *-Algebras, Vol. II *algebras. (Encyclopedia of mathematics and its applications: Vol. 79) Cambridge University Press, 2001.

[8] R.T. Powers, Self-adjoint algebras of unbounded operators. Comm. Math. Phys., 21 (1971) 85-124.

[9] C.E. Rickart, General theory of Banach algebras, (The University Series in Higher Mathematics ). Van Nostrand, 1960.

[10] K. Schmüdgen, Unbounded Operator Algebras and Representation Theory, (Operator Theory: Advances and Applications, Vol.37). Birkhäuser - Verlag, 1990. 
[11] C. Trapani, *-Representations, seminorms and structure properties of normed quasi *algebras. Studia Math., 186 (2008) 47-75.

Dipartimento di Matematica e Informatica, Università degli Studi di Palermo, Via Archirafi 34, I-90123 Palermo, Italy

E-mail address: bellomonte@math.unipa.it 